\title{
Photorefractive properties of lithium and copper in-diffused lithium niobate crystals
}

\author{
Jörg Imbrock, Albert Wirp, Detlef Kip, and Eckhard Krätzig \\ Universität Osnabrück, Fachbereich Physik, D-49069 Osnabrück, Germany \\ Dirk Berben \\ Physikalisches Institut, Universität Bonn, Wegelerstrasse 8, D-53115 Bonn, Germany
}

Received September 21, 2001; revised manuscript received March 20, 2002

\begin{abstract}
Near-stoichiometric copper-doped lithium niobate crystals are fabricated by in-diffusion of thin layers of evaporated copper and a subsequent vapor transport equilibration treatment. The crystals are heated in a Li-rich atmosphere to increase the Li content. To determine the photorefractive properties, holographic as well as electrical measurements are performed. Saturation values of the refractive-index changes $\Delta n_{\mathrm{S}}$, bulk photovoltaic current densities $j_{\mathrm{phv}}$, photoconductivities $\sigma_{\mathrm{ph}}$, and holographic sensitivities $S$ are measured for light intensities up to $10^{4} \mathrm{~W} / \mathrm{m}^{2}$. Comparison with experimental data of congruent crystals indicates that the specific photoconductivity is 15 times larger after a vapor transport equilibration treatment. The specific bulk photovoltaic coefficient $\beta^{*}$ is 2 times larger, refractive-index changes are 7 times smaller, and the holographic sensitivity is up to 4 times larger. (c) 2002 Optical Society of America

OCIS codes: $160.5320,210.2860,090.7330$.
\end{abstract}

\section{INTRODUCTION}

Lithium niobate $\left(\mathrm{LiNbO}_{3}\right)$ is one of the most promising photorefractive materials for applications like wavelength filters ${ }^{1}$ and holographic data storage. ${ }^{2}$ The photorefractive effect is based upon the buildup of inhomogeneous space-charge fields under an inhomogeneous illumination with light. The space-charge fields modulate the refractive index through the electro-optic effect. In this way, thick phase holograms can be recorded in $\mathrm{LiNbO}_{3}$. Normally, $\mathrm{LiNbO}_{3}$ is grown from the congruent melt where the $\mathrm{Li}$ concentration $c_{\mathrm{Li}}$ is not equal to the $\mathrm{Nb}$ concentration $c_{\mathrm{Nb}}$. The relative Li content $c_{\mathrm{Li}} /\left(c_{\mathrm{Li}}+c_{\mathrm{Nb}}\right)$ of congruently melting $\mathrm{LiNbO}_{3}$ is $\sim 48.4 \%$, which is much smaller than the stoichiometric composition. ${ }^{3}$ The photorefractive properties strongly depend on defects and impurities of the crystal. Doping with, e.g., iron or copper leads to an enlarged photorefractive effect. ${ }^{4}$ Iron- and copper-doped congruent $\mathrm{LiNbO}_{3}$ crystals have been examined in detail, and their holographic properties are now well understood. It turns out that the dynamic range, which is determined by the saturation value of refractiveindex changes $\Delta n_{\mathrm{S}}$, is limited at a value of $\sim 7 \times 10^{-4}$, which cannot be further enhanced by increasing the doping level. ${ }^{5,6}$ Larger doping concentrations lead to an increased photoconductivity and dark conductivity.

The fundamental limit of the dynamic range of $\mathrm{LiNbO}_{3}$ is a great disadvantage for applications such as holographic data storage. Furthermore, congruent $\mathrm{LiNbO}_{3}$ has a large response time and a low sensitivity compared with other holographic storage materials like, e.g., photopolymers. These properties can be influenced by different dopants and thermal treatments in a certain range, but an enhancement of the photoconductivity is always related to a decrease of the dynamic range. However, be- sides varying the concentration of extrinsic defects, it is possible to influence the intrinsic defects. Due to the $\mathrm{Li}$ deficit of congruent $\mathrm{LiNbO}_{3}$, there exist a lot of $\mathrm{Li}$ vacancies and $\mathrm{Nb}_{\mathrm{Li}}\left(\mathrm{Nb}\right.$ on $\mathrm{Li}$ site) antisite defects. ${ }^{7}$ The concentration of vacancies and antisites can be decreased with the help of a VTE (vapor transport equilibration) technique. ${ }^{8-10} \quad \mathrm{LiNbO}_{3}$ crystals are heated in a Li-rich atmosphere, which leads to a diffusion of $\mathrm{Li}$ ions into the sample. The Li ions are placed on vacancies or displace $\mathrm{Nb}_{\mathrm{Li}}$ ions, and the result is an increased Li content. Our interest is focused on the influence of a VTE treatment on the photorefractive properties of copper-doped $\mathrm{LiNbO}_{3}$ because, up to now, the holographic properties of nearstoichiometric $\mathrm{LiNbO}_{3}$ are not examined in detail. $\mathrm{LiNbO}_{3}$ can easily be doped with copper by an in-diffusion technique because the diffusion constant for copper is more than 500 times higher than the diffusion constant for iron. ${ }^{11}$

The charge-transport process in copper-doped $\mathrm{LiNbO}_{3}$ at usual intensities of $\mathrm{cw}$ lasers $\left(\leqslant 10^{5} \mathrm{~W} / \mathrm{m}^{2}\right)$ can be well understood in terms of a one-center model: under an inhomogeneous illumination with visible light, electrons are excited from filled $\mathrm{Cu}^{+}$traps to the conduction band. The electrons are redistributed and finally retrapped by $\mathrm{Cu}^{2+}$. When no external electrical field is applied, there are two main contributions to the current density $j$ : a diffusion and a photovoltaic current. Diffusion effects depend only on the recording geometry and on temperature. In transmission geometry, the diffusion part can normally be neglected, and the photovoltaic effect is the dominant process. The one-center model yields the following relations ${ }^{12}: j_{\mathrm{phv}} \propto I c_{\mathrm{Cu}^{+}}, \sigma_{\mathrm{ph}} \propto I\left(c_{\mathrm{Cu}^{+}} / c_{\mathrm{Cu}^{2+}}\right)$, and $\Delta n_{\mathrm{S}} \propto E_{\mathrm{SC}}=j_{\mathrm{phv}} / \sigma_{\mathrm{ph}} \propto c_{\mathrm{Cu}^{2+}}$. Here $j_{\mathrm{phv}}$ denotes the bulk photovoltaic current density, $\sigma_{\mathrm{ph}}$ is the photoconduc- 
tivity, $\Delta n_{\mathrm{S}}$ is the saturation value of refractive-index changes, $E_{\mathrm{SC}}$ is the space-charge field, $c_{\mathrm{Cu}^{+}}$and $c_{\mathrm{Cu}^{2+}}$ are the concentrations of filled and empty traps, respectively, and $I$ is the average light intensity inside the crystal.

We have fabricated ${ }^{6,11}$ lithium niobate crystals with different copper concentrations with an in-diffusion technique. A subsequent VTE treatment produces nearstoichiometric $\mathrm{LiNbO}_{3}: \mathrm{Cu}$. All parameters that are relevant for holographic data storage are measured. We present how the dynamic range $\Delta n_{\mathrm{S}}$, the photoconductivity $\sigma_{\mathrm{ph}}$, and the holographic sensitivity $S$ are influenced by the increased Li concentration.

\section{EXPERIMENTAL METHODS}

\section{A. Samples}

The samples are cut from 1-mm-thick 3-in (7.5-cm) $\mathrm{LiNbO}_{3}$ wafers, and they have typical dimensions of $x \times y \times z=5 \mathrm{~mm} \times 1 \mathrm{~mm} \times 6 \mathrm{~mm}$. Onto one surface normal to the $y$ axis a copper layer is vacuum deposited. The layer thickness varies between $100 \mathrm{~nm}$ and $1000 \mathrm{~nm}$. After the deposition, the crystals are annealed at $1000^{\circ} \mathrm{C}$ for $150 \mathrm{~h}$ in air. Peithmann et al. ${ }^{6}$ have demonstrated that, after this treatment, the whole copper is distributed homogeneously inside the crystal. The notation of the crystals indicates the thickness of the deposited copper layer, e.g., a crystal where a copper layer of $100 \mathrm{~nm}$ has been in-diffused, and is named Cu100VTE.

After this in-diffusion process, the crystals have been VTE-treated. Therefore a powder of $\mathrm{Nb}_{2} \mathrm{O}_{5}$ and $\mathrm{Li}_{2} \mathrm{CO}_{3}$ is sintered for $3 \mathrm{~h}$ to ensure a good homogeneity. The powder containing $65-\mathrm{mol} \% \mathrm{Li}_{2} \mathrm{O}$ was put together with the copper-doped lithium niobate crystals into an oven.
We have performed two treatments: first, we have heated the weaker-doped crystals for $150 \mathrm{~h}$ at $1100{ }^{\circ} \mathrm{C}$. The second treatment has taken $200 \mathrm{~h}$ at $1100{ }^{\circ} \mathrm{C}$, and it included the more highly doped crystals. To determine the stoichiometry of the crystals, we have treated simultaneously an undoped wafer piece in the oven. The absorption edge of this sample was shifted to a smaller wavelength, which is the consequence of an increased $\mathrm{Li}$ content in the crystal. ${ }^{13,14}$ The relative $\mathrm{Li}$ content $c_{\mathrm{Li}} /\left(c_{\mathrm{Li}}+c_{\mathrm{Nb}}\right)$ is increased from $48.4 \%$ to approximately $(49.8 \pm 0.1) \%$.

After the VTE treatment, all samples were cut into two pieces $a$ and $b$. One piece (b) from each sample was thermally reduced in a vacuum atmosphere $(0.3 \mathrm{~Pa})$ at $650{ }^{\circ} \mathrm{C}$ for $12 \mathrm{~h}$ to increase the $c_{\mathrm{Cu}^{+}} / c_{\mathrm{Cu}^{2}}$ ratio. To determine the copper concentration, we have measured the absorption coefficient of the crystals in the visible and infrared spectral region with a CARY 500 spectrometer. The absorption constants $\alpha_{477 \mathrm{~nm}}^{\mathrm{o}}$ and $\alpha_{1040 \mathrm{~nm}}^{\mathrm{o}}$ for ordinarily polarized light at $477 \mathrm{~nm}$ and $1040 \mathrm{~nm}$, respectively, yield the $\mathrm{Cu}^{+}$and $\mathrm{Cu}^{2+}$ concentrations ${ }^{4,15}$ :

$$
\begin{gathered}
c_{\mathrm{Cu}^{+}}=2.0 \times 10^{21} \mathrm{~m}^{-2} \alpha_{477 \mathrm{~nm}}^{\mathrm{o}}, \\
c_{\mathrm{Cu}^{2+}}=6.8 \times 10^{22} \mathrm{~m}^{-2} \alpha_{1040 \mathrm{~nm}}^{0} .
\end{gathered}
$$

In addition, electron-paramagnetic-resonance (EPR) measurements have been performed with three samples (Cu400VTEb, Cu500VTEb, and Cu1000VTEa) to determine the content of $\mathrm{Cu}^{2+}$. These measurements yield $\mathrm{Cu}^{2+}$ concentrations that are $\sim 30 \%$ larger than the concentrations determined by absorption measurements, which indicates that the factor $6.8 \times 10^{22} \mathrm{~m}^{-2}$ in Eq. (2) might be too small. Table 1 summarizes the used lithium

Table 1. Notation, Thickness, Deposited Thickness of Copper Layer, Total Copper Concentration Calculated from the Thickness of the Deposited layer and from Absorption Measurements, and

\begin{tabular}{|c|c|c|c|c|c|}
\hline Notation & $\begin{array}{l}\text { Thickness } \\
\text { [mm] }\end{array}$ & $\begin{array}{c}\text { Cu Layer } \\
\text { Thickness } \\
\text { [nm] }\end{array}$ & $\begin{array}{c}c_{\mathrm{Cu}}\left[10^{23} \mathrm{~m}^{-3}\right] \\
\text { by Cu Layer } \\
\text { Thickness }\end{array}$ & $\begin{array}{c}c_{\mathrm{Cu}}\left[10^{23} \mathrm{~m}^{-3}\right] \\
\text { by } \\
\text { Absorption }\end{array}$ & $\begin{array}{c}c_{\mathrm{Cu}^{+}}\left[10^{23} \mathrm{~m}^{-3}\right] \\
\text { by } \\
\text { Absorption }\end{array}$ \\
\hline Cu100VTEa & 0.97 & 100 & 85 & 64 & 12 \\
\hline Cu100VTEb & 0.98 & 100 & 85 & 64 & 36 \\
\hline Cu200VTEa & 0.98 & 200 & 170 & 156 & 30 \\
\hline Cu200VTEb & 0.98 & 200 & 170 & 156 & 57 \\
\hline Cu300VTEa & 0.98 & 300 & 255 & 232 & 48 \\
\hline Cu300VTEb & 0.98 & 300 & 255 & 232 & 76 \\
\hline Cu400VTEa & 0.98 & 400 & 340 & 329 & 67 \\
\hline $\mathrm{Cu} 400 \mathrm{VTEb}$ & 0.98 & 400 & 340 & 329 & 102 \\
\hline Cu500VTEa & 0.91 & 500 & 425 & 260 & 59 \\
\hline Cu500VTEb & 0.91 & 500 & 425 & 260 & 110 \\
\hline Cu600VTEa & 0.90 & 600 & 510 & 314 & 79 \\
\hline Cu600VTEb & 0.89 & 600 & 510 & 314 & 122 \\
\hline Cu800VTEa & 0.87 & 800 & 680 & 369 & 97 \\
\hline Cu800VTEb & 0.87 & 800 & 680 & 369 & 136 \\
\hline Cu1000VTEa & 0.91 & 1000 & 850 & 396 & 100 \\
\hline Cu1000VTEb & 0.91 & 1000 & 850 & 396 & 131 \\
\hline
\end{tabular}
Concentration of $\mathrm{Cu}^{+}$of the Used Lithium Niobate Crystals ${ }^{a}$

${ }^{a}$ The number in the notation denotes the deposited thickness of the copper layer. Crystals whose names are ending with "b" have been thermally reduced in a vacuum atmosphere $(0.3 \mathrm{~Pa})$ for $12 \mathrm{~h}$ at temperature of $650{ }^{\circ} \mathrm{C}$ to increase the $\mathrm{Cu}^{+}$concentration. The VTE treatment was performed at $1100{ }^{\circ} \mathrm{C}$ in a lithium-rich atmosphere with a powder containing 65 -mol.\% $\mathrm{Li}_{2} \mathrm{O}$. The first VTE treatment was performed with the weaker-doped crystals (Cu100VTEa ... Cu400VTEb), and it has taken $150 \mathrm{~h}$. The second VTE treatment with the higher-doped crystals (Cu500VTEa ... Cu1000VTEb) has taken $200 \mathrm{~h}$. 
niobate crystals with the measured copper concentrations.

\section{B. Holographic Characterization}

Holographic measurements are performed with a twobeam interference setup (Fig. 1). Green light $(\lambda$ $=514 \mathrm{~nm}$, ordinary polarization) of an argon-ion laser is split into two beams (R1 and R2) of equal intensity up to $10^{4} \mathrm{~W} / \mathrm{m}^{2}$. These two recording beams are superimposed inside the lithium niobate crystal, which is mounted in front of an aperture (diameter $=1 \mathrm{~mm}$ ). The interference pattern has a fringe spacing of $\Lambda=0.8$ $\mu \mathrm{m}$, and the grating vector $\mathbf{K}$ is lying parallel to the crystal's $c$ axis. The hologram can be probed during recording with a weak beam of a helium neon $(\mathrm{He}-\mathrm{Ne})$ laser $(\lambda=633 \mathrm{~nm}$, ordinary polarization) that is Bragg matched. While recording a hologram, the transmitted intensity $I_{\mathrm{t}}$ and the diffracted intensity $I_{\mathrm{d}}$ of the probe laser beam is measured. These intensities yield the diffraction efficiency $\eta=I_{\mathrm{d}} /\left(I_{\mathrm{d}}+I_{\mathrm{t}}\right)$. Using Kogelnik's formula, ${ }^{16}$

$$
\eta=\sin ^{2}\left(\frac{\pi \Delta n d}{\lambda \cos \Theta}\right)
$$

we calculate the refractive-index change $\Delta n$. Here, $d$ denotes the crystal thickness, $\lambda$ the vacuum wavelength, and $\Theta$ is half of the angle between the recording beams inside the crystal. When the recorded hologram has reached its maximum refractive-index change $\Delta n_{\mathrm{S}}$, one recording beam is blocked, and the hologram is read out with the remaining beam. The resulting refractive-index change is compared with that one calculated from the diffracted light of the probe laser to ensure a proper alignment of the probe laser beam. Additionally, we can read a hologram with the $\mathrm{He}-\mathrm{Ne}$ laser beam and rotate the crystal slightly to check whether the probe laser beam is correctly Bragg matched.

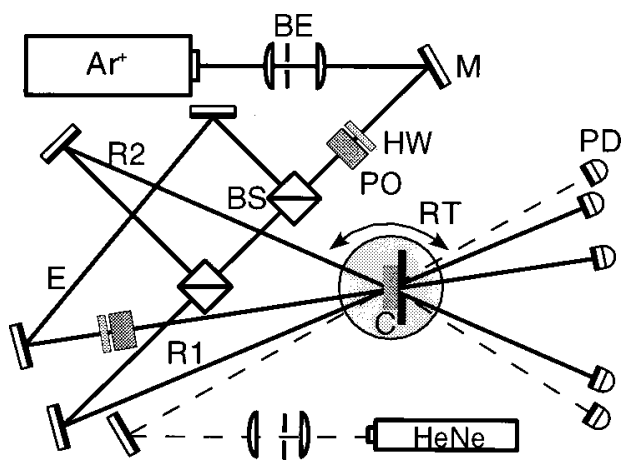

Fig. 1. Schematic drawing of the holographic setup. Two ordinarily polarized recording beams (R1 and R2) of an Ar-ion laser $(\lambda=514 \mathrm{~nm})$ are superimposed. The $\mathbf{K}$ vector of the interference pattern is lying parallel to the crystal's $c$ axis. During hologram recording and erasure, the grating can be probed with red light $(\lambda=633$, ordinary polarization) of a He-Ne laser. OffBragg erasure is achieved by illuminating the crystal with a different beam (E). C, crystal on rotation table (RT); M, mirror: $\mathrm{BS}$, beam splitter; HW, half-wave plate; PO, polarizer; PD, photodiode.
A hologram can be erased with a third laser beam offBragg $(\lambda=514 \mathrm{~nm}$, ordinary polarization). The temporal behavior of hologram recording and erasure follows a monoexponential law:

$$
\begin{aligned}
& \Delta n=\Delta n_{S}\left[1-\exp \left(-t / \tau_{\mathrm{r}}\right)\right], \text { for recording, } \\
& \Delta n=\Delta n_{S} \exp \left(-t / \tau_{\mathrm{e}}\right), \text { for erasure. }
\end{aligned}
$$

The photoconductivity can be obtained either from the recording time constant $\tau_{\mathrm{r}}$ or from the erasure time constant $\tau_{\mathrm{e}}$ by $\sigma_{\mathrm{ph}}=\epsilon \epsilon_{0} / \tau_{\mathrm{e}, \mathrm{r}}$, with the dielectric constant $\epsilon=28$ (Ref. 17) and the permittivity of free space $\epsilon_{0}$. All measurements are carried out under short-circuited conditions that are achieved by contacting the crystals edges with silver-paste electrodes. Since crystals with different $\mathrm{Cu}^{+}$concentration exhibit different absorption constants in the visible, an averaged intensity is calculated with the formula

$$
I=I_{\mathrm{in}} \frac{1-R}{\alpha^{\mathrm{o} d}} \frac{1-\exp \left(-\alpha^{\mathrm{o}} d\right)}{1-R \exp \left(-\alpha^{\mathrm{o}} d\right)} .
$$

Here $R$ denotes the reflectivity, and $I_{\text {in }}$ is the intensity of the incident light.

Some crystals show light-induced absorption changes $\alpha_{\mathrm{li}}$ in the red spectral region during illumination with green light. When the crystal is illuminated with light of the erasing beam, the absorption coefficient for red light increases and reaches its saturation value in less than one second. The saturation value of light-induced absorption change is determined by measuring the transmitted red light of the probe laser while illuminating with green light:

$$
\alpha_{\mathrm{li}}=1 / d \ln \left[I_{\mathrm{t}}(0) / I_{\mathrm{t}}(t)\right] .
$$

Here $I_{\mathrm{t}}(0)$ is the transmitted probe laser intensity without green illumination, and $I_{\mathrm{t}}(t)$ denotes the transmitted intensity after switching on the green light.

Bulk photovoltaic current densities ${ }^{18}$ are measured conventionally. By means of a grating monochromator, the wavelength of the light of a xenon arc lamp can be adjusted between $400 \mathrm{~nm}$ and $600 \mathrm{~nm}$. The light is ordinarily polarized, and its intensity can be varied with neutral-density filters. When the crystal is illuminated homogeneously, a bulk photovoltaic current flows along the $c$ axis. We measure this current by contacting the surfaces perpendicular to the $c$ axis with silver-paste electrodes and connecting the electrodes with a highsensitivity electrometer. A detailed description of the setup is given in Ref. 19 .

\section{EXPERIMENTAL RESULTS}

\section{A. Samples}

After the copper in-diffusion and the subsequent VTE treatment, all crystals are polished to optical quality, and the spectral dependence of the absorption constant is measured. The absorption spectrum shows two shoulders at $400 \mathrm{~nm}$ and $1040 \mathrm{~nm}$, respectively (Fig. 2). A thermal reduction (Cu200VTEb) increases $\alpha_{400 \mathrm{~nm}}$ and decreases $\alpha_{1040 \mathrm{~nm}}$. Most of the crystals exhibit a very large absorption at $400 \mathrm{~nm}$ that cannot be measured with thick samples. Hence we have determined the $\mathrm{Cu}^{+}$concentra- 


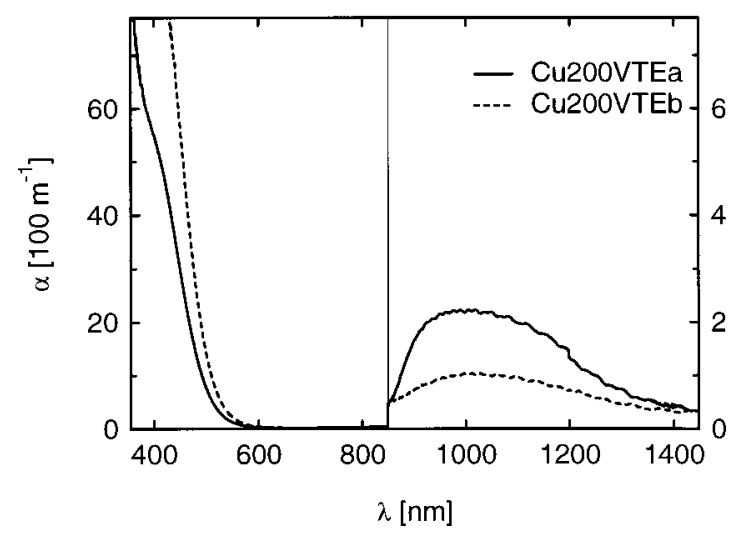

Fig. 2. Absorption coefficient $\alpha$ versus wavelength $\lambda$ for ordinarily polarized light. The reduced crystal Cu200VTEb exhibits a larger absorption at $400 \mathrm{~nm}$, which is proportional to the amount of $\mathrm{Cu}^{+}$. The absorption at $\sim 1000 \mathrm{~nm}$ is proportional to the concentration of $\mathrm{Cu}^{2+}$.

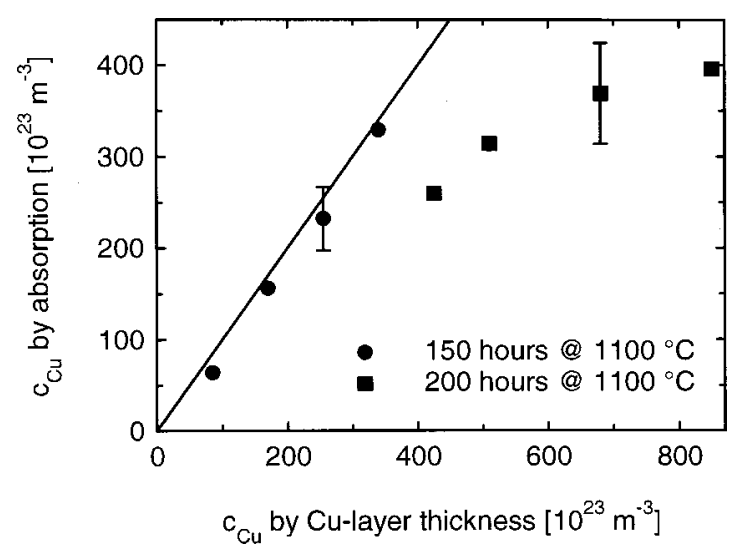

Fig. 3. Total copper concentration $c_{\mathrm{Cu}}$ that follows from absorption measurements as a function of the copper concentration determined by the evaporated copper-layer thickness. Crystals with a thickness of the evaporated copper layer larger than 500 $\mathrm{nm}$ (squares) have been annealed $50 \mathrm{~h}$ longer in the lithium-rich atmosphere than the other samples.

tion from the absorption coefficient at $477 \mathrm{~nm}$ [Eq. (1)]. The total copper concentration $c_{\mathrm{Cu}}$ can be calculated from the evaporated copper-layer thickness: a thickness of $100 \mathrm{~nm}$ corresponds to a copper concentration of 8.5 $\times 10^{24} \mathrm{~m}^{-3}$ in our 1 -mm-thick samples. The calculated copper concentration is compared with the concentration determined by the absorption measurements (Fig. 3). After the weaker-doped crystals have been VTE treated (circles), nearly all evaporated copper is distributed inside the samples. The second VTE treatment was performed with the higher-doped crystals (squares), and it has taken $50 \mathrm{~h}$ longer than the first series. During this treatment, part of the copper diffuses out of the samples because the absorption measurements yield much smaller concentrations than expected, and a thin copper layer can be seen on the inner walls of the crucible.

\section{B. Saturation Values of Refractive-Index Changes}

The variation of the refractive-index changes with the concentration of deep traps $c_{\mathrm{Cu}^{2+}}$ is presented in Fig. 4. The maximum refractive-index change $\Delta n_{\mathrm{S}}$ depends linearly on $c_{\mathrm{Cu}^{2+}}$ with $\Delta n_{\mathrm{S}} / c_{\mathrm{Cu}^{2+}}=5 \times 10^{-30} \mathrm{~m}^{3}$. Up to a concentration of $c_{\mathrm{Cu}}=3.0 \times 10^{25} \mathrm{~m}^{-3}$, we cannot observe any saturation behavior of $\Delta n_{\mathrm{S}}$. In some crystals, $\Delta n_{\mathrm{S}}$ varies with the intensity $I$ of recording light (Fig. 5). Crystals containing a small amount of $\mathrm{Cu}^{2+}$ ions show an enlarged $\Delta n_{\mathrm{S}}$ with increasing recording intensity. For higher intensities, $\Delta n_{\mathrm{S}}$ tends to saturate, and this saturation value is again larger, if the $\mathrm{Cu}^{2+}$ concentration is small. Crystals with a $\mathrm{Cu}^{2+}$ concentration larger than $1.0 \times 10^{25} \mathrm{~m}^{-3}$ do not show any variations of $\Delta n_{\mathrm{S}}$ with recording intensity. To compare the values of $\Delta n_{\mathrm{S}}$ of all crystals (Fig. 4), we have always taken the intensityindependent part $\Delta n_{\mathrm{S}}^{0}$ (Fig. 5).

The intensity dependence of $\Delta n_{\mathrm{S}}$ is correlated with the appearance of light-induced absorption changes $\alpha_{\mathrm{li}}$. Under illumination with green light, the absorption at 633 nm increases (Fig. 6). This increase of absorption is also detectable at $514 \mathrm{~nm}$, but $\alpha_{1 \mathrm{i}}^{514 \mathrm{~nm}}$ is much smaller than $\alpha_{\mathrm{li}}^{633 \mathrm{~nm}}$. With increasing intensity of the green light, $\alpha_{\mathrm{li}}$ reaches a maximum. Crystals that exhibit large saturation values of $\alpha_{\mathrm{li}}$ also show a strong dependence of $\Delta n_{\mathrm{S}}$ on the recording intensity.

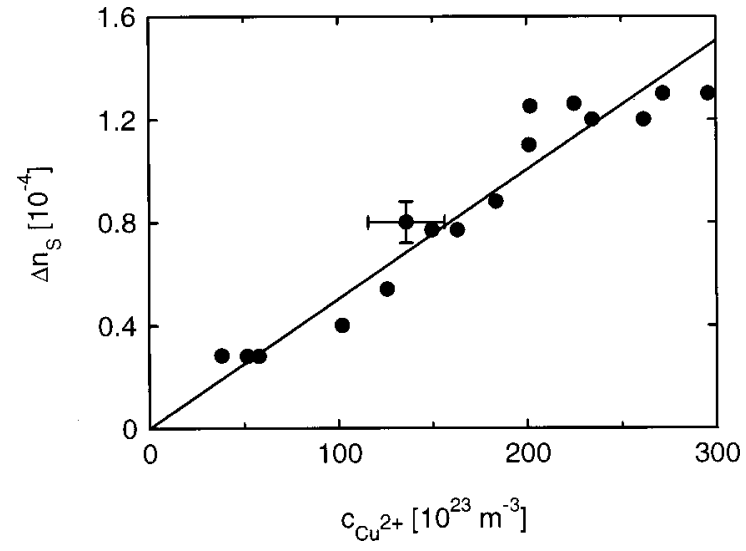

Fig. 4. Effect of trap concentration $c_{\mathrm{Cu}^{2}}$ on saturation values of refractive-index change $\Delta n_{\mathrm{S}}$. Because some crystals exhibit an intensity dependence of $\Delta n_{\mathrm{S}}$ (see Fig. 5), we have compared always the intensity-independent part $\Delta n_{\mathrm{S}}^{0}$. The line is a linear fit to the measured values.

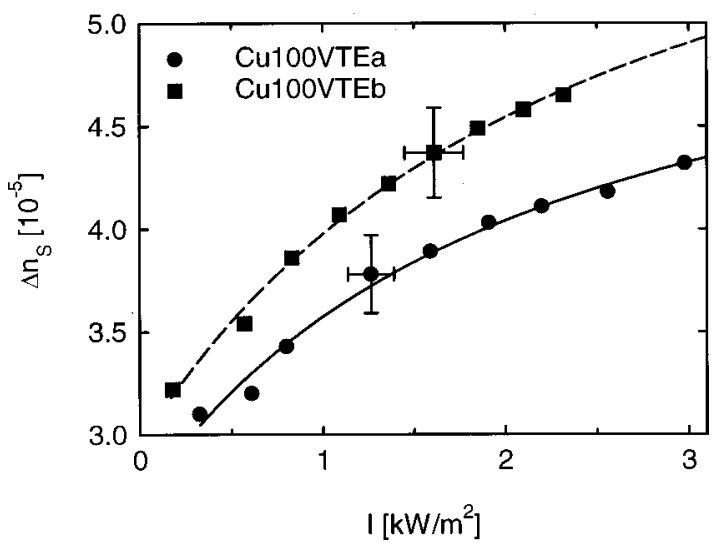

Fig. 5. Variation of saturation values of refractive-index change $\Delta n_{\mathrm{S}}$ with intensity of recording light $I$ for $\lambda=514 \mathrm{~nm}$. In crystals with a small concentration of $\mathrm{Cu}^{2+}$ ions, $\Delta n_{\mathrm{S}}$ is increasing with increasing recording intensity. Crystals containing more than $10^{25} \mathrm{~m}^{-3} \mathrm{Cu}^{2+}$ do not show any variations of $\Delta n_{\mathrm{S}}$ with recording intensity. The curves are fits according to $\Delta n_{\mathrm{S}}(I)$ $=\Delta n_{\mathrm{S}}^{0}+\Delta n_{\mathrm{S}}^{\max } \times I /($ const. $+I)$. 


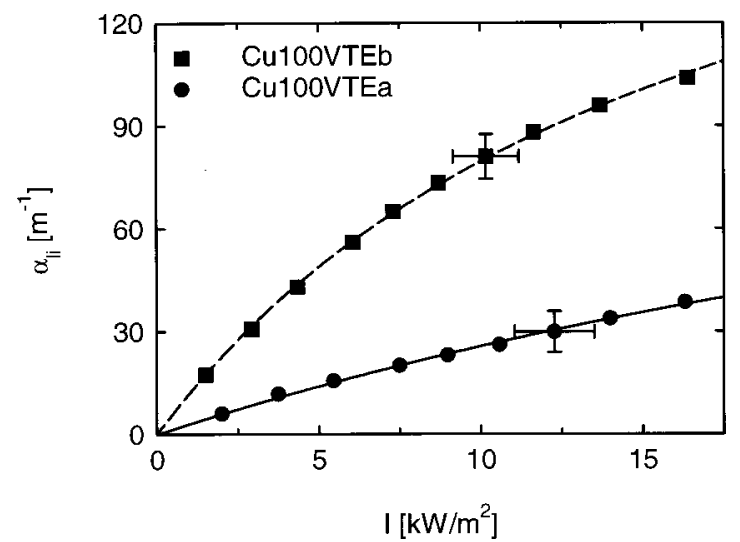

Fig. 6. Light-induced absorption change $\alpha_{\text {li }}$ for ordinarily polarized red $(\lambda=633 \mathrm{~nm})$ probe light versus green $(\lambda=514 \mathrm{~nm})$ pump intensity $I$. The curves are fits according to $\alpha_{\mathrm{li}}(I)$ $=\alpha_{\mathrm{li}}^{\max } \times I /($ const. $+I)$.

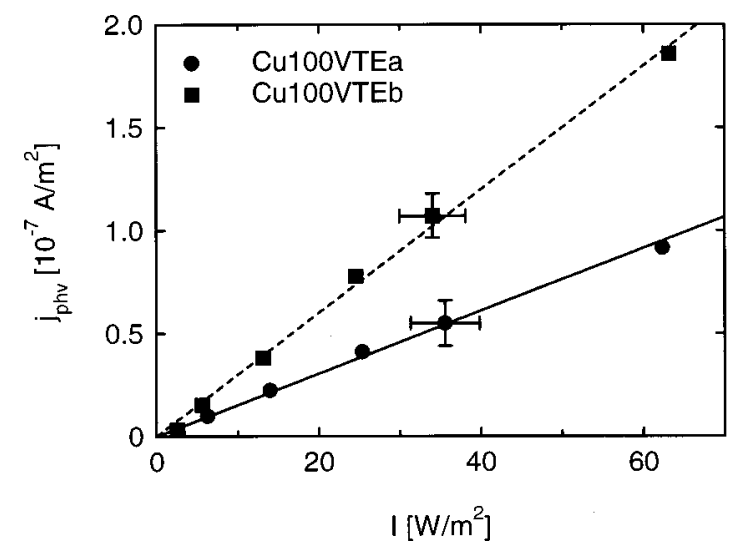

Fig. 7. Bulk photovoltaic current density $j_{\text {phv }}$ versus light intensity for $\lambda=520 \mathrm{~nm}$. The lines are linear fits to the measured data.

\section{Bulk Photovoltaic Effect}

Bulk photovoltaic currents are measured for different light intensities at wavelengths between $400 \mathrm{~nm}$ and 600 $\mathrm{nm}$ and for different concentrations of filled traps $c_{\mathrm{Cu}^{+}}$. The bulk photovoltaic current density $j_{\text {phv }}$ depends always linearly on light intensity (Fig. 7). The ratio $j_{\mathrm{phv}} / I$ grows linearly with $c_{\mathrm{Cu}^{+}}$, and the slope of the linear fit in Fig. 8 yields the specific photovoltaic coefficient $\beta^{*}=j_{\mathrm{phv}} /$ $\left(I c_{\mathrm{Cu}^{+}}\right)=1.1 \times 10^{-33} \mathrm{~m}^{3} / \mathrm{V}$ at $\lambda=520 \mathrm{~nm}$. We have proved for all samples that $\beta^{*}$ does not depend on the total copper concentration $c_{\mathrm{Cu}}$. The spectral dependence of $\beta^{*}$ is presented in Fig. 9. The shape of the curve is comparable to the absorption spectrum (Fig. 2). A maximum of $\beta^{*}=1.8 \times 10^{-32} \mathrm{~m}^{3} / \mathrm{V}$ is observed at $\sim 400 \mathrm{~nm}$, which is 16 times larger than $\beta_{514 \mathrm{~nm}}^{*}$. We can compare the measured values with $\beta^{*}$ of congruent crystals containing the same amount of copper. It turns out that after the VTE treatment, $\beta^{*}$ has doubled.

\section{Photoconductivity}

The variation of the photoconductivity $\sigma_{\mathrm{ph}}$ with light intensity is presented in Fig. 10 with the samples Cu100VTEa and Cu100VTEb. The photoconductivity increases in a wide range linearly with intensity. Reduced samples show a larger photoconductivity because $\sigma_{\mathrm{ph}}$ is proportional to the $c_{\mathrm{Cu}^{+}} / c_{\mathrm{Cu}^{2+}}$ ratio (Fig. 11). The specific photoconductivity $f=\sigma_{\mathrm{ph}} /\left[I\left(c_{\mathrm{Cu}^{+}} / c_{\mathrm{Cu}^{2+}}\right)\right]=2.1 \times 10^{-14}$ $\mathrm{m} / \mathrm{V}^{2}$ does not depend on the total copper concentration. In comparison with weakly-copper-doped congruent crystals, $f$ is 15 times larger due to the VTE treatment. A typical time constant $\tau$ for recording or erasing a holo-

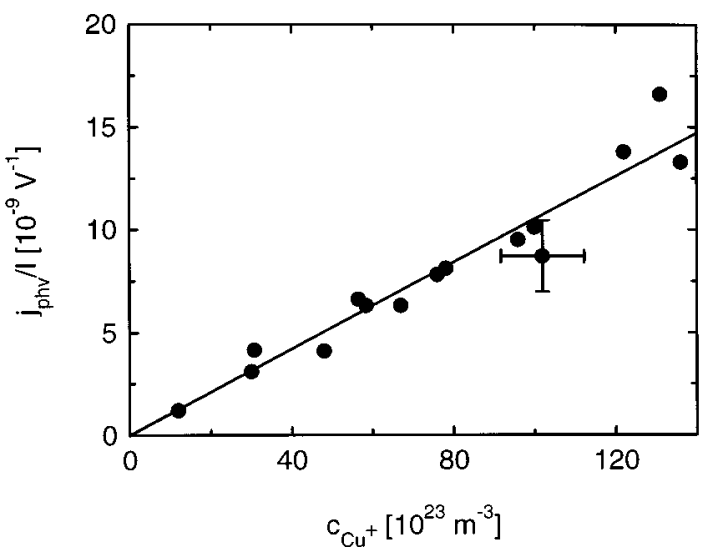

Fig. 8. Variation of the normalized bulk photovoltaic current density $j_{\mathrm{phv}} / I$ with the concentration of $\mathrm{Cu}^{+}$ions. The line is a linear fit to the data.

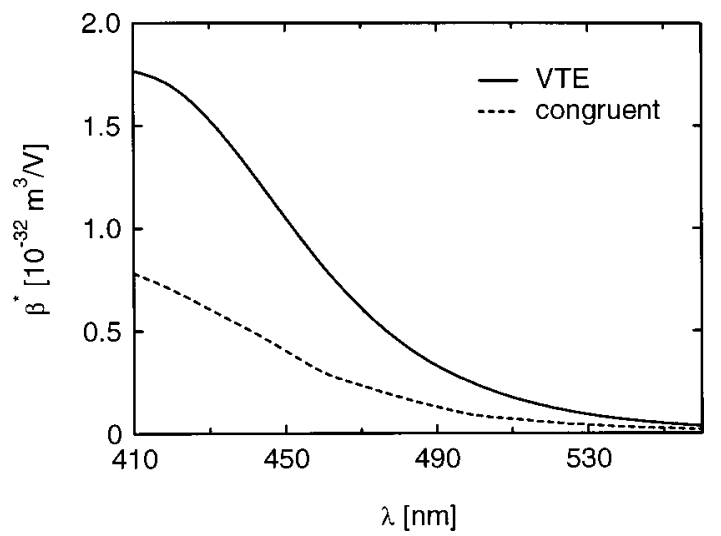

Fig. 9. Spectral dependence of specific photovoltaic coefficient $\beta^{*}=\left(j_{\mathrm{phv}} / I\right) / c_{\mathrm{Cu}^{+}}$for congruent and VTE-treated crystals. VTE-treated crystals show a 2 times-larger specific photovoltaic coefficient than congruent crystals.

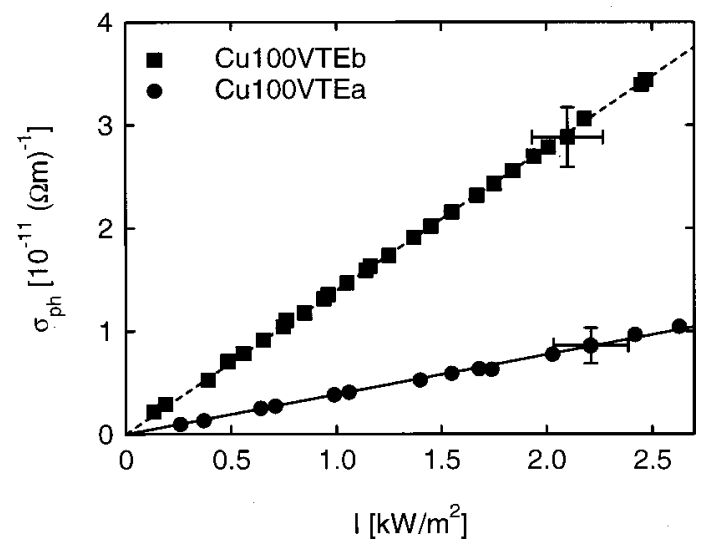

Fig. 10. Photoconductivity $\sigma_{\mathrm{ph}}$ versus light intensity $I$ for $\lambda$ $=514 \mathrm{~nm}$. The photoconductivity depends always linearly on the light intensity. 


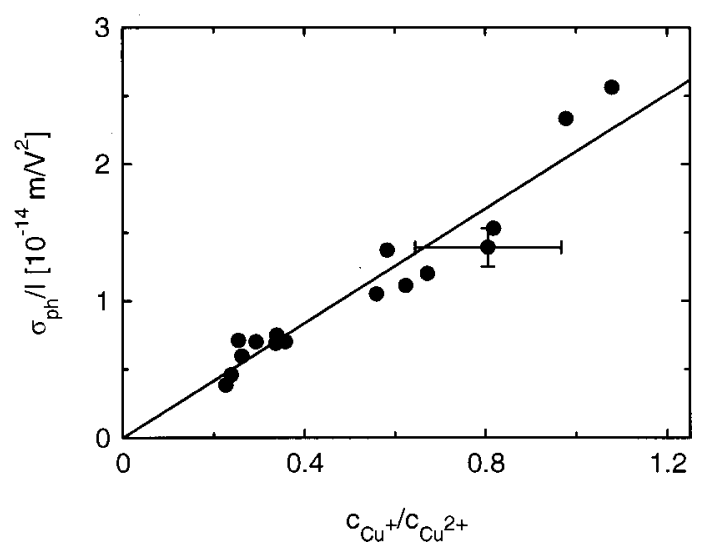

Fig. 11. Variation of the normalized photoconductivity $\sigma_{\mathrm{ph}} / I$ with the $c_{\mathrm{Cu}^{+}} / c_{\mathrm{Cu}^{2}}$ ratio. The normalized photoconductivity is increasing linearly with increasing $c_{\mathrm{Cu}^{+}} / c_{\mathrm{Cu}^{2}}$ ratio.

gram is $24 \mathrm{~s}\left(I=1 \mathrm{~kW} / \mathrm{m}^{2}, c_{\mathrm{Cu}^{+}} / c_{\mathrm{Cu}^{2+}}=0.5\right)$, and even recording times of only $1 \mathrm{~s}$ can be realized.

Aside from the photoconductivity, one could think that also the dark conductivity is influenced by the VTE treatment. First temperature-dependent dark-decay measurements yield a hologram lifetime of about one year at room temperature. This value is of the order of as-grown copper-doped $\mathrm{LiNbO}_{3}$ crystals.

\section{DISCUSSION}

Diffusion of evaporated copper layers into $\mathrm{LiNbO}_{3}$ wafers and a subsequent VTE treatment is an easy way to produce doped near-stoichiometric $\mathrm{LiNbO}_{3}$ crystals. Crystals with thicknesses up to $1 \mathrm{~mm}$ can be fabricated in moderate times. Thicker crystals need larger diffusion and annealing times to distribute the copper and the lithium homogeneously. Comparing the copper concentrations determined by absorption measurements and those calculated from the deposited copper layer thickness reveals that the copper concentration is limited by the duration of the VTE treatment. After a VTE treatment of $150 \mathrm{~h}$ almost all copper is still distributed inside the samples. After $200 \mathrm{~h}$, some copper is diffused out of the samples. This out-diffusion depends only on the time of the VTE treatment. After the in-diffusion procedure of the copper, we get copper concentrations up to 14.5 $\times 10^{25} \mathrm{~m}^{-3}{ }^{6}$ We think that the decreased $\mathrm{Cu}$ concentration after the VTE treatment is due to a replacement of the $\mathrm{Cu}$ ions by $\mathrm{Li}$ ions that diffuse into the sample. After the $\mathrm{Li}$ in-diffusion, the maximum copper concentration in our crystals is $4.0 \times 10^{25} \mathrm{~m}^{-3}$. So, the VTE treatment has a great influence on the copper content, but we cannot say in what way the longer VTE treatment influences the lithium content. However, it seems that the prolonged VTE treatment does not further influence the photorefractive properties.

We can understand the results on the photorefractive properties in terms of a one-center model, if we calculate the space-charge field $E_{\mathrm{SC}}$ from the results of the direct measurements of the photovoltaic current density $j_{\mathrm{phv}}$ and of the photoconductivity $\sigma_{\mathrm{ph}}$. The saturation value of the space-charge field $E_{\mathrm{SC}}=j_{\mathrm{phv}} / \sigma_{\mathrm{ph}}$ modulates the refractive-index change $\Delta n_{\mathrm{S}}=0.5 n_{\mathrm{eff}}^{3} r_{\mathrm{eff}} E_{\mathrm{SC}}$ through the electro-optic effect ( $n_{\text {eff }}$, effective refractive index; $r_{\text {eff }}$ effective electro-optic coefficient). We have already proved that the photovoltaic current density $j_{\mathrm{phv}}$ can be described by $j_{\mathrm{phv}}=\beta^{*} c_{\mathrm{Cu}^{+}} I$. The photoconductivity depends on the concentration of electrons in the conduction band $n_{\mathrm{e}}, \sigma_{\mathrm{ph}}=e \mu_{\mathrm{e}} n_{\mathrm{e}}\left(\mu_{\mathrm{e}}\right.$, mobility of electrons). For this parameter $n_{\mathrm{e}}$, the one-center model yields $n_{\mathrm{e}}$ $=(s q / \gamma) I\left(c_{\mathrm{Cu}^{+}} / c_{\mathrm{Cu}^{2+}}\right)$. Here, the absorption cross section, $s$, and the quantum efficiency, $q$, of the $\mathrm{Cu}^{+}$ions depend on the wavelength of the light. The coefficient $\gamma$ describes the recombination of an electron from the conduction band with a $\mathrm{Cu}^{2+}$ ion. Using this relation, we can express the specific photoconductivity as $f=\sigma_{\mathrm{ph}} /$ $\left[I\left(c_{\mathrm{Cu}^{+}} / c_{\mathrm{Cu}^{2+}}\right)\right]=e \mu_{\mathrm{e}} s q / \gamma$. Now, the space-charge field can be described by $f$ and $\beta^{*}$ with $E_{\mathrm{SC}}=\left(\beta^{*} / f\right) c_{\mathrm{Cu}^{2+}}$. This leads to the expression $\Delta n_{\mathrm{S}} / c_{\mathrm{Cu}^{2+}}=0.5 n_{\mathrm{eff}}^{3} r_{\mathrm{eff}}\left(\beta^{*} / f\right)$. With $\beta^{*}=1.1 \times 10^{-33} \mathrm{~m}^{3} / \mathrm{V}, f=2.1 \times 10^{-14} \mathrm{~m} / \mathrm{V}^{2}, n_{\text {eff }}$ $=2.33$ (Ref. 20), and $r_{\text {eff }}=1.1 \times 10^{-11} \mathrm{~m} / \mathrm{V}$ (Ref. 21), we get $\Delta n_{\mathrm{S}} / c_{\mathrm{Cu}^{2+}}=3.6 \times 10^{-30} \mathrm{~m}^{3}$. This value fits very well with the measured value of $5.0 \times 10^{-30} \mathrm{~m}^{3}$, if we consider that $\beta^{*}$ was determined at $520 \mathrm{~nm}$. Therefore $\beta^{*}$ at $514 \mathrm{~nm}$ should be slightly larger.

It has been observed in congruent copper-doped $\mathrm{LiNbO}_{3}$ that $\Delta n_{\mathrm{S}}$ has a fundamental limit. ${ }^{16} \Delta n_{\mathrm{S}}$ cannot exceed the value $7 \times 10^{-4}$. Trap densities $c_{\mathrm{Cu}^{2}}$ larger than $4.0 \times 10^{25} \mathrm{~m}^{-3}$ lead to an increased photo and dark conductivity. In our near-stoichiometric crystals we have achieved a maximum $\Delta n_{\mathrm{S}}$ of $1.2 \times 10^{-4}$ with a $\mathrm{Cu}^{2+}$ concentration of $3.0 \times 10^{25} \mathrm{~m}^{-3}$ (Fig. 4). Up to this trap density, we cannot see any saturation for $\Delta n_{\mathrm{S}}$. Thus it should be possible to achieve a large $\Delta n_{\mathrm{S}}$ if we further increase the copper concentration.

The appearance of light-induced absorption changes cannot be understood with only one photorefractive center. We have to take into account that a second center is involved in the light-induced charge transport. The $\mathrm{LiNbO}_{3}$ crystals contain not only extrinsic defects $\left(\mathrm{Cu}^{+/ 2+}\right)$ but also intrinsic defects, namely, niobium ions on a lithium site $\left(\mathrm{Nb}_{\mathrm{Li}}^{5+}\right)$. Under illumination with green light, electrons can be excited to the conduction band as well as to $\mathrm{Nb}_{\mathrm{Li}}^{5+}$, thus forming $\mathrm{Nb}_{\mathrm{Li}}^{4+}$ polarons. These polarons absorb light of the near-infrared spectral region, ${ }^{22}$ which leads to a light-induced absorption change at $\lambda$ $=633 \mathrm{~nm}$. The absorption change $\alpha_{\mathrm{li}}$ is the larger the more $\mathrm{Nb}_{\mathrm{Li}}^{5+}$ centers can be populated. The saturation value of the concentration of $\mathrm{Nb}_{\mathrm{Li}}^{4+}$ polarons under green illumination depends on the total amount of $\mathrm{Nb}_{\mathrm{Li}}^{5+}$ ions and on the lifetime of the $\mathrm{Nb}_{\mathrm{Li}}^{4+}$ polarons because in the dark all electrons from $\mathrm{Nb}_{\mathrm{Li}}^{4+}$ recombine with deep traps $\left(\mathrm{Cu}^{2+}\right)$. This polaron lifetime is larger in samples with a lower $\mathrm{Cu}^{2+}$ content. The VTE treated crystals exhibit polaron lifetimes up to a few seconds, which is very large in comparison with congruent crystals, where the lifetime is of the order of microseconds only. ${ }^{23}$ The concentration of $\mathrm{Nb}_{\mathrm{Li}}^{5+}$ ions is decreased during the VTE treatment because Li diffuses to their places. Therefore after the VTE treatment, the concentration of $\mathrm{Nb}_{\mathrm{Li}}^{5+}$ ions is small but the lifetime is very large due to an enhanced activation energy. $^{23}$ This is the reason that, even with low intensities, a large number of polarons can be created. 
It is known that the $\mathrm{Nb}_{\mathrm{Li}}^{4+}$ polarons can contribute to the photorefractive effect. ${ }^{24}$ Depending on the $\mathrm{Nb}_{\mathrm{Li}}^{4+}$ concentration $c_{\mathrm{N} b_{\mathrm{Li}}^{4+}}$ and on the light intensity $I$, the photovoltaic current density can be increased because of an additional contribution from the polarons: $j=j_{\mathrm{Cu}}+j_{\mathrm{Nb}}$, with $j_{\mathrm{Nb}}=\beta_{\mathrm{Nb}}^{*} I c_{\mathrm{Nb}_{\mathrm{Li}}^{4+}}$. The concentration $c_{\mathrm{Nb}_{\mathrm{Li}}^{4+}}$ varies with light intensity in the same way as $\alpha_{\text {li }}$ depends on the light intensity. This means that $j_{\mathrm{Nb}}$ grows quadratically with $I$ if the intensities are small. As the photoconductivity varies always linearly with $I$ (Fig. 10), the resulting $\Delta n_{\mathrm{S}}$ increases with increasing light intensity (Fig. 5).

The specific photovoltaic coefficient $\beta_{\mathrm{Nb}}^{*}$ of the polarons is very large at $\sim 780 \mathrm{~nm}$, but at $514 \mathrm{~nm}$ the influence on the current density is detectable, too. The large photovoltaic effect in the near-infrared and the long lifetimes of the polarons in VTE-treated samples can also be used for nonvolatile holographic storage..$^{25,26}$ The crystal is illuminated homogeneously with green or blue light, and holograms are recorded with infrared light, for example, $\lambda$ $=785 \mathrm{~nm}$. These holograms can be read nondestructively with infrared light only, because infrared light cannot excite electrons from $\mathrm{Cu}^{+}$ions. We have already tested this method successfully with our nearstoichiometric copper-doped $\mathrm{LiNbO}_{3}$ crystals, but the results have not yet been published.

The enhancement of $\beta^{*}$ due to the VTE treatment also increases the holographic sensitivity $S$, which is defined as $S=\partial \sqrt{\eta} /\left.\partial t\right|_{t=0} /(I d)$. For hologram recording we get $S=\sqrt{\eta} /(\tau I d) \propto j_{\text {phv }} / I \propto \beta^{*}$. Since $\beta^{*}$ is doubled, the sensitivity is doubled, too. The sensitivity is also proportional to the amount of $\mathrm{Cu}^{+}$. From our measurements we can derive the following relation: $S / c_{\mathrm{Cu}^{+}}=2.6$ $\times 10^{-27} \mathrm{~m}^{4} / \mathrm{J}$. The sample Cu1000VTEa $\left(c_{\mathrm{Cu}^{+}}=100\right.$ $\times 10^{23} \mathrm{~m}^{-3}$ ) shows a sensitivity of $2.1 \mathrm{~cm} / \mathrm{J}$, which agrees very well with the calculated value of $2.6 \mathrm{~cm} / \mathrm{J}$. Normally $S$ does not depend on the recording intensity, but crystals that exhibit an intensity-dependent $\Delta n_{\mathrm{S}}$, e.g., Cu100VTEb, also show an intensity-dependent sensitivity. The sensitivity of the crystal Cu100VTEb $\left(c_{\mathrm{Cu}^{+}}\right.$ $=38 \times 10^{23} \mathrm{~m}^{-3}$ ) increases with increasing recording intensity and has a maximum value of $1.9 \mathrm{~cm} / \mathrm{J}$. This value is 2 times larger than the calculated one. Therefore the sensitivity of VTE-treated crystals with a small content of deep traps can be up to 4 times larger than the sensitivity of congruent crystals. The fact that $\beta^{*}$ is twice as large as before the treatment is quite interesting, because the VTE treatment is often discussed as a method to reduce the optical damage, which means to reduce the photorefractive effect. This can be achieved by a smaller photovoltaic current $j$ and/or by a larger photoconductivity $\sigma_{\mathrm{ph}}$. However, we have seen that in our copper-doped crystals a VTE treatment increases the photovoltaic current, but the photoconductivity is increased much more, which finally results in a smaller spacecharge field and refractive-index change, respectively.

The photoconductivity is one order of magnitude larger than before the VTE treatment. The origin of the increased $\sigma_{\mathrm{ph}}$ can be explained in three different ways. Regarding the specific photoconductivity $f=e \mu_{\mathrm{e}} s q / \gamma$, it is possible that $\mu_{\mathrm{e}}$ is increased, the product $s q$ is increased, or $\gamma$ is decreased. To determine the copper concentration from absorption measurements, we have used the absorption cross sections that are valid for congruent crystals. As these measurements deliver reliable values, we can assume that $s q$ remains unchanged after the VTE treatment. The electron mobility $\mu_{\mathrm{e}}$ can be increased since the concentration of shallow traps $\left(\mathrm{Nb}_{\mathrm{Li}}^{5+}\right)$ is small after the VTE treatment. But it is also possible that the recombination coefficient $\gamma$ has changed. After the indiffusion of copper, the copper ions are especially placed on $\mathrm{Li}$ sites because in congruent $\mathrm{LiNbO}_{3}$, many $\mathrm{Li}$ sites are not occupied. ${ }^{27}$ During the VTE treatment, lithium ions diffuse into the sample and are placed either on $\mathrm{Li}$ vacancies, on $\mathrm{Li}$ sites where $\mathrm{Nb}$ has been located, or on $\mathrm{Li}$ sites where $\mathrm{Cu}$ has been located. Some copper diffuses out of the sample during the treatment, and some copper ions migrate from $\mathrm{Li}$ sites to $\mathrm{Nb}$ sites. $\mathrm{Cu}^{2+}$ ions that are placed on $\mathrm{Nb}^{5+}$ sites might have different capture cross sections than $\mathrm{Cu}^{2+}$ ions that are placed on $\mathrm{Li}^{+}$sites. Thus the recombination coefficient $\gamma$ may be decreased after the VTE treatment. The assumption that copper ions occupy different places in congruent and nearstoichiometric crystals, respectively, is substantiated by EPR measurements. The EPR spectrum of nearstoichiometric lithium niobate crystals is sharper and more symmetrical than that one of congruent crystals. ${ }^{28}$ To distinguish whether a change of $\mu_{\mathrm{e}}$ or $\gamma$ is responsible for the large photoconductivity, Hall measurements could be more informative.

\section{CONCLUSIONS}

Doping of $\mathrm{LiNbO}_{3}$ by in-diffusion of thin copper layers and a subsequent VTE treatment produces nearstoichiometric photorefractive samples. The copper concentration is limited by the duration and temperature of the VTE treatment as copper diffuses out of the samples during this treatment. Most of the measured dependences for the saturation values of refractive-index changes $\Delta n_{\mathrm{S}}$, photoconductivities $\sigma_{\mathrm{ph}}$, and bulk photovoltaic current densities $j_{\text {phv }}$ can be understood in terms of a one-center model. The appearance of light-induced absorption changes and an intensity-dependent $\Delta n_{\mathrm{S}}$ indicates that also polarons $\left(\mathrm{Nb}_{\mathrm{Li}}^{4+}\right)$ are involved in the lightinduced charge transport. After the VTE treatment, the photoconductivity is 15 times increased, and the specific bulk photovoltaic coefficient $\beta^{*}$ is 2 times enlarged. The result is a 7-times-smaller $\Delta n_{\mathrm{S}}$ but also an increased holographic sensitivity $S$. An improvement of the dynamic range $\left(\Delta n_{\mathrm{S}}\right)$ should be possible if the copper concentration could be increased furthermore.

\section{ACKNOWLEDGMENT}

We thank M. Meyer for performing the EPR measurements and K. Peithmann for fruitful discussions. Financial support of the Deutsche Forschungsgemeinschaft (SFB 255, A6) and of the Bundesministerium für Bildung und Forschung (grant 13N8076) is gratefully acknowledged. D. Berben thanks the Deutsche Forschungsgemeinschaft for financial support (Bu 913/8-1). 


\section{REFERENCES}

1. S. Breer, H. Vogt, I. Nee, and K. Buse, "Low-crosstalk WDM by Bragg diffraction from thermally fixed reflection holograms in lithium niobate," Electron. Lett. 34, 2418-2421 (1998).

2. H. J. Coufal, D. Psaltis, and G. T. Sincerbox, eds., Holographic Data Storage (Springer-Verlag, Berlin, 2000), Vol. 76.

3. P. F. Bordui, R. G. Norwood, C. D. Bird, and G. D. Calvert, "Compositional uniformity in growth and poling of largediameter lithium niobate crystals," J. Cryst. Growth 113, 61-68 (1991).

4. E. Krätzig and R. Orlowski, "Light-induced charge transport in doped $\mathrm{LiNbO}_{3}$ and $\mathrm{LiTaO}_{3}$," Ferroelectrics 27, 241244 (1980).

5. K. Peithmann, A. Wiebrock, and K. Buse, "Photorefractive properties of highly-doped lithium niobate crystals in the visible and near-infrared," Appl. Phys. B 68, 777-784 (1999).

6. K. Peithmann, J. Hukriede, K. Buse, and E. Krätzig, "Photorefractive properties of $\mathrm{LiNbO}_{3}$ crystals doped by copper diffusion," Phys. Rev. B 61, 4615-4620 (2000).

7. S. C. Abrahams and P. Marsh, "Defect structure dependence on composition in lithium niobate," Acta Crystallogr., Sect. B 42, 61-68 (1986).

8. D. H. Jundt, M. M. Fejer, and R. L. Byer, "Optical properties of lithium-rich lithium niobate fabricated by vapor transport equilibration," IEEE J. Quantum Electron. 26, 135-138 (1990).

9. P. F. Bordui, R. G. Norwood, D. H. Jundt, and M. M. Fejer, "Preparation and characterization of off-congruent lithium niobate crystals," J. Appl. Phys. 71, 875-879 (1992).

10. D. H. Jundt, M. M. Fejer, R. G. Norwood, and P. F. Bordui, "Composition dependence of lithium diffusivity in lithium niobate at high temperature," J. Appl. Phys. 72, 3468-3473 (1992).

11. J. Hukriede, B. Gather, D. Kip, and E. Krätzig, "Copper diffusion into lithium niobate," Phys. Status Solidi A 172, R3 (1999).

12. K. Buse, "Light-induced charge transport processes in photorefractive crystals I: Models and experimental methods," Appl. Phys. B 64, 273-291 (1997).

13. L. Kovacs, G. Ruschhaupt, K. Polgar, G. Corradi, and M. Wöhlecke, "Composition dependence of the ultraviolet absorption edge in lithium niobate," Appl. Phys. Lett. 70, 2801-2803 (1997).
14. D. Redfield and W. J. Burke, "Optical absorption edge of $\mathrm{LiNbO}_{3}$," J. Appl. Phys. 45, 4566-4571 (1974).

15. K. Buse, S. Breer, K. Peithmann, S. Kapphan, M. Gao, and E. Krätzig, "Origin of thermal fixing in photorefractive lithium niobate crystals," Phys. Rev. B 56, 1225-1235 (1997).

16. H. Kogelnik, "Coupled wave theory for thick hologram gratings,” Bell Syst. Tech. J. 48, 2909-2947 (1969).

17. A. Mansingh and A. Dhar, "The AC conductivity and dielectric constant of lithium niobate single crystals," J. Phys. D 18, 2059-2071 (1985).

18. A. M. Glass, D. von der Linde, and T. J. Negran, "Highvoltage bulk photovoltaic effect and the photorefractive process in $\mathrm{LiNbO}_{3}, "$ Appl. Phys. Lett. 25, 233-235 (1974).

19. K. Buse, U. van Stevendaal, R. Pankrath, and E. Krätzig, "Light-induced charge transport properties of $\mathrm{Sr}_{0.61} \mathrm{Ba}_{0.39} \mathrm{Nb}_{2} \mathrm{O}_{6}$ crystals,” J. Opt. Soc. Am. B 13, 14611467 (1996).

20. R. S. Weis and T. K. Gaylord, "Lithium niobate: summary of physical properties and crystal structure," Appl. Phys. A 37, 191-203 (1985).

21. K. Onuki, N. Uchida, and T. Saku, "Interferometric method for measuring electro-optic coefficients in crystals," J. Opt. Soc. Am. 62, 1030-1032 (1972).

22. K. L. Sweeney and L. E. Halliburton, "Oxygen vacancies in lithium niobate," Appl. Phys. Lett. 43, 336-338 (1983).

23. D. Berben, K. Buse, S. Wevering, P. Herth, M. Imlau, and T. Woike, "Lifetime of small polarons in iron-doped lithiumniobate crystals," J. Appl. Phys. 87, 1034-1041 (2000).

24. F. Jermann and J. Otten, "Light-induced charge transport in $\mathrm{LiNbO}_{3}: \mathrm{Fe}$ at high light intensities," J. Opt. Soc. Am. B 10, 2085-2092 (1993).

25. H. Guenther, R. Macfarlane, Y. Furukawa, K. Kitamura, and R. Neurgaonkar, "Two-color holography in reduced near-stoichiometric lithium niobate," Appl. Opt. 37, 76117623 (1998).

26. L. Hesselink, S. Orlov, A. Liu, A. Akella, D. Lande, and R. Neurgaonkar, "Photorefractive materials for nonvolatile volume holographic data storage," Science 282, 1089-1094 (1998)

27. O. F. Schirmer, O. Thiemann, and M. Wöhlecke, "Defects in $\mathrm{LiNbO}_{3}$-I. Experimental aspects," J. Phys. Chem. Solids 52, 185-189 (1991).

28. G. I. Malovichko, V. G. Grachev, E. P. Kokanyan, O. F. Schirmer, K. Betzler, B. Gather, F. Jermann, S. Klauer, U. Schlarb, and M. Wöhlecke, "Characterization of stoichiometric $\mathrm{LiNbO}_{3}$ grown from melts containing $\mathrm{K}_{2} \mathrm{O}$," Appl. Phys. A 56, 103-108 (1993). 